\title{
Quantitative Comparison of Sinc-Approximating Kernels for Medical Image Interpolation
}

\author{
Erik H. W. Meijering, Wiro J. Niessen, Josien P. W. Pluim, and \\ Max A. Viergever \\ Image Sciences Institute, Utrecht University \\ Heidelberglaan 100, 3584 CX Utrecht, The Netherlands \\ \{erik, wiro, josien, $\max \}$ @isi.uu.nl \\ http://www.isi.uu.nl
}

\begin{abstract}
Interpolation is required in many medical image processing operations. From sampling theory, it follows that the ideal interpolation kernel is the sinc function, which is of infinite extent. In the attempt to obtain practical and computationally efficient image processing algorithms, many sinc-approximating interpolation kernels have been devised. In this paper we present the results of a quantitative comparison of 84 different sinc-approximating kernels, with spatial extents ranging from 2 to 10 grid points in each dimension. The evaluation involves the application of geometrical transformations to medical images from different modalities (CT, MR, and PET), using the different kernels. The results show very clearly that, of all kernels with a spatial extent of 2 grid points, the linear interpolation kernel performs best. Of all kernels with an extent of 4 grid points, the cubic convolution kernel is the best ( $28 \%-75 \%$ reduction of the errors as compared to linear interpolation). Even better results (44\% - 95\% reduction) are obtained with kernels of larger extent, notably the Welch, Cosine, Lanczos, and Kaiser windowed sinc kernels. In general, the truncated sinc kernel is one of the worst performing kernels.
\end{abstract}

\section{Introduction}

Interpolation of sampled data is required in a variety of medical image processing operations, such as rotation, translation, deformation, or magnification, which are frequently applied for registration or visualization purposes. In many applications, it is of paramount importance to limit as much as possible the grey-value errors introduced by interpolation. For example, in image registration, interpolation errors may introduce artifacts in the optimization cost function, which may lead to registration errors [1]. Furthermore, it has been pointed out that, especially in the case of functional images, interpolation errors may affect the interpretation of longitudinal studies [2]. It has also been shown that the errors made by interpolation kernels influence the results of measurements carried out in maximum intensity projection images [3].

Whereas from sampling theory it follows that the ideal interpolation kernel is the sinc function, it is not the ideal kernel from an implementational point of 
view, since this function is of infinite extent and has a very low rate of decay. In the attempt to obtain practical and computationally efficient image processing algorithms, many sinc-approximating interpolation kernels have been devised. However, an extensive quantitative comparison of the performance of these kernels when using them to apply geometrical transformations to medical images, has never been described. The purpose of this paper is to present the results of such a comparison.

\section{Sinc-Approximating Kernels}

In this section we briefly present the sinc-approximating kernels most frequently encountered in the literature. These can be divided into piecewise polynomial kernels and windowed sinc kernels.

\subsection{Piecewise Polynomial Kernels}

A frequently used approach to obtain a sinc-approximating kernel is to model the shape of the sinc kernel by piecewise polynomials. The simplest approach in this respect is to use zeroth order polynomials, resulting in the so-called nearest neighbor kernel:

$$
h_{N N}(x)=\left\{\begin{array}{l}
1,-\frac{1}{2} \leqslant x<\frac{1}{2} \\
0, x<-\frac{1}{2} \vee x \geqslant \frac{1}{2}
\end{array}\right.
$$

Higher order piecewise polynomial kernels can be written in the form [4]:

$$
h(x)= \begin{cases}a_{0 j}+a_{1 j}|x|+\ldots+a_{n j}|x|^{n} & , j \leqslant|x|<j+1 \\ 0 & , m \leqslant|x|\end{cases}
$$

where $n$ is the order of the polynomials, $j=0,1, \ldots, m-1$, the parameter $m \in \mathbb{N}_{\backslash\{0\}}$ determines the extent of the kernel, and $n$ and $m$ are related by $n=2 m-1$. The $(n+1) m$ coefficients $a_{i j}$ can be solved by imposing constraints on the polynomials, derived from the shape of the sinc kernel [4].

For $n=1$, Eq. (2) boils down to the linear interpolation kernel, $h_{\text {Lin }}$. The resulting kernels for $n>1$ can be shown to be functions of a free parameter, $\alpha$. In order to obtain a unique value for $\alpha$, one additional constraint needs to be imposed. In the literature on cubic convolution $(n=3)$, several constraints have been proposed [5]: (i) The slope constraint, which implies that $\alpha$ is chosen such that the slope of the kernel equals the slope of the sinc function at $x=1$. This value is denoted $\alpha_{\varsigma}$. (ii) The continuity constraint. For $n>1$, the piecewise polynomial kernels are elements of $C^{n-2}$. The continuity constraint implies that $\alpha$ is chosen such that the $(n-1)$ th-order derivative of the kernel is continuous at $x=1$. The resulting value is denoted $\alpha_{\sim}$. (iii) The flatness constraint, which implies that $\alpha$ is chosen such that the Fourier spectrum of the kernel, $\tilde{H}(f)$, is flat at $f=0$. This value of $\alpha$ is denoted $\alpha_{b}$. These three constraints can also be applied to the higher order schemes. The resulting values of $\alpha$ for the cubic $\left(h_{C u b}\right)$, quintic $\left(h_{Q u i}\right)$, septic $\left(h_{S e p}\right)$, and nonic $\left(h_{N o n}\right)$ piecewise polynomial interpolation kernels, are presented in Table 1. 


\begin{tabular}{lrrr}
\hline \hline Kernel & $\alpha_{\varsigma}$ & \multicolumn{1}{c}{$\alpha_{\sim}$} & $\alpha_{b}$ \\
\hline$h_{\text {Cub }}$ & -1 & $-\frac{3}{4}$ & $-\frac{1}{2}$ \\
$h_{\text {Qui }}$ & $\frac{11}{96}$ & $\frac{1}{13}$ & $\frac{3}{64}$ \\
$h_{\text {Sep }}$ & $-\frac{1027}{452574}$ & $-\frac{3133}{2275008}$ & $-\frac{71}{83232}$ \\
$h_{\text {Non }}$ & $\frac{34814699}{2509872453120}$ & $\frac{17671607}{2324998440576}$ & $\frac{3829}{788235264}$ \\
\hline \hline
\end{tabular}

Table 1. Values of the free parameter $\alpha$ for the cubic $\left(h_{C u b}\right)$, quintic $\left(h_{Q u i}\right)$, septic $\left(h_{S e p}\right)$, and nonic $\left(h_{N o n}\right)$ piecewise polynomial interpolation kernels, resulting from the slope constraint $\left(\alpha_{\varsigma}\right)$, continuity constraint $\left(\alpha_{\sim}\right)$, and flatness constraint $\left(\alpha_{b}\right)$.

\subsection{Windowed Sinc Kernels}

Another approach to obtain a sinc-approximating kernel is to multiply the sinc function with a window function of limited extent:

$$
h(x)=\omega(x) \operatorname{sinc}(x), \quad \text { with } \omega(x)= \begin{cases}w(x), 0 \leqslant|x|<m \\ 0 \quad, m \leqslant|x|\end{cases}
$$

where $\omega: \mathbb{R} \rightarrow \mathbb{R}$ is the window function, and $w: \mathbb{R} \rightarrow \mathbb{R}$ determines the shape of the window in the interval $(-m, m)$, with $m \in \mathbb{N}_{\backslash\{0\}}$. The window functions which were used in the quantitative comparison described in the next section are listed in Table 2.

\section{Quantitative Comparison}

The sinc-approximating kernels presented in the previous section were quantitatively compared by using them to apply several geometrical transformations to a number of medical images, and by analyzing the resulting interpolation errors in the transformed images. In this section we present the evaluation strategy and the results.

\subsection{Evaluation Strategy}

Medical images were obtained from a collection of 3-D brain datasets from three different modalities, viz., computed tomography (CT), magnetic resonance imaging (MR; T1-weighted), and positron emission tomography (PET). From every subset (modality), we selected five images. The five CT images were of size $512 \times 512$ times $28,29,33,30$, and 28 voxels, respectively, all with a voxel size of $0.654 \times 0.654 \times 4.0 \mathrm{~mm}^{3}$. The five T1-weighted MR images were all of size $256 \times 256 \times 26$ voxels, with a voxel size of $1.25 \times 1.25 \times 4.0 \mathrm{~mm}^{3}$. The five PET images were of size $128 \times 128 \times 15$ voxels, one with a voxel size of 


\begin{tabular}{ll}
\hline \hline Window & Definition \\
\hline Bartlett & $w_{\text {Bar }} \triangleq 1-\frac{|x|}{m}$ \\
Blackman & $w_{\text {Bla }} \triangleq 0.42+0.50 \cos \left(\frac{\pi x}{m}\right)+0.08 \cos \left(\frac{2 \pi x}{m}\right)$ \\
Blackman-Harris & $w_{B H a} \triangleq 0.42323+0.49755 \cos \left(\frac{\pi x}{m}\right)+0.07922 \cos \left(\frac{2 \pi x}{m}\right)$ \\
Bohman & $w_{\text {Boh }} \triangleq\left(1-\frac{|x|}{m}\right) \cos \left(\frac{\pi x}{m}\right)+\frac{1}{\pi} \sin \left(\frac{\pi|x|}{m}\right)$ \\
Cosine & $w_{\text {Cos }} \triangleq \cos \left(\frac{\pi x}{2 m}\right)$ \\
Hamming & $w_{\text {Ham }} \triangleq 0.54+0.46 \cos \left(\frac{\pi x}{m}\right)$ \\
Hann & $w_{\text {Han }} \triangleq 0.5+0.5 \cos \left(\frac{\pi x}{m}\right)$ \\
Kaiser & $w_{\text {Kai }} \triangleq \frac{I_{0}(\beta)}{I_{0}(\alpha)}, \beta=\alpha \sqrt{1-\left(\frac{x}{m}\right)^{2}}$ \\
Lanczos & $w_{\text {Lan }} \triangleq \operatorname{sinc}\left(\frac{\pi x}{m}\right)$ \\
Rectangular & $w_{\text {Rec }} \triangleq 1$ \\
Welch & $w_{\text {Wel }} \triangleq 1-\frac{x^{2}}{m^{2}}$ \\
\hline \hline
\end{tabular}

Table 2. Window functions and their definitions. Throughout this paper, the corresponding kernels are given the same subscript. In the definition of the Kaiser window, $\alpha \in \mathbb{R}^{+}$is a free parameter, for which we used values of 5.0,6.0, 7.0, and 8.0. $I_{0}$ is the zeroth order modified Bessel function of the first kind, which can accurately be approximated by using its series expansion. For details, see Harris [6] or Wolberg [5].

$1.94 \times 1.94 \times 8.0 \mathrm{~mm}^{3}$, and the others with a voxel size of $2.59 \times 2.59 \times 8.0 \mathrm{~mm}^{3}$. In order to be able to study the performance of the interpolation kernels in different slice directions, we selected, for each of the 3-D images, one transversal and one sagittal slice. This resulted in a total of 30 different 2-D test-images (see Fig. 1 for examples).

The test-images were subjected to several geometrical transformations, including rotations and subpixel translations, as these are frequently required in (multimodality) registration. In the rotation experiments, the test-images were successively rotated over $0.7^{\circ}, 3.2^{\circ}, 6.5^{\circ}, 9.3^{\circ}, 12.1^{\circ}, 15.2^{\circ}, 18.4^{\circ}, 21.3^{\circ}, 23.7^{\circ}$, $26.6^{\circ}, 29.8^{\circ}, 32.9^{\circ}, 35.7^{\circ}, 38.5^{\circ}, 41.8^{\circ}$, and $44.3^{\circ}$, which adds up to a total of $360^{\circ}$. In the subpixel translation experiments, the images were successively shifted over $0.01,0.04,0.07,0.11,0.15,0.18,0.21,0.24,0.26,0.29,0.32,0.35$, $0.39,0.43,0.46$, and 0.49 pixels, which adds up to a total of 4.0 pixels. For every test-image, both experiments were carried out for all interpolation kernels. Of the two families described in Section 2, we used all kernels for which $m \leqslant 5$, which amounts to a total of 84 kernels. In order to avoid border problems, the test-images were mirrored around the borders in each dimension.

For every combination of test-image, experiment (rotation or translation), and interpolation kernel, the root-mean-square error (RMSE) of the grey-values in the processed image was computed. Since in these experiments the grid points 

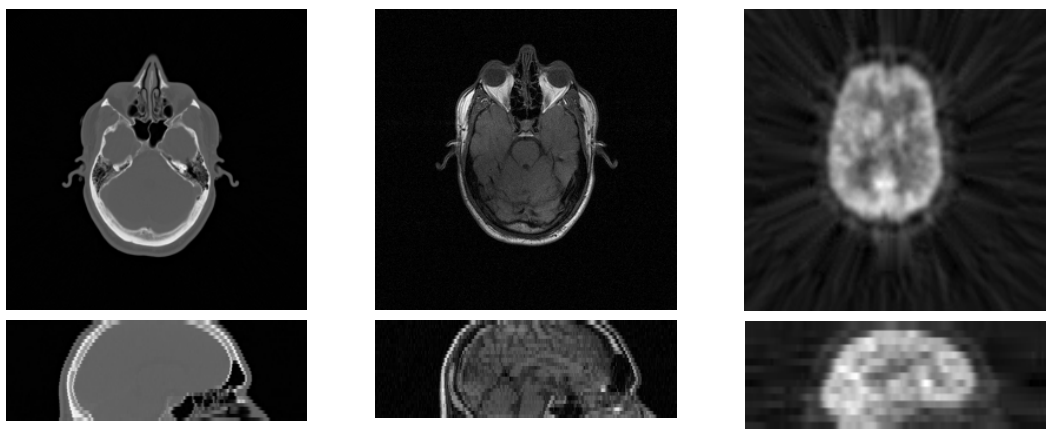

Fig. 1. Examples of the medical test-images used in the experiments. Top row (left-to-right): a transversal slice of a 3-D CT, MR-T1, and PET dataset, respectively. Bottom row: sagittal slices of the same datasets. Note that for display purposes, the images of the sagittal slices shown in this figure were scaled, thus correcting for the voxel anisotropy. Nearest neighbor interpolation was used for this purpose.

of the processed images coincide with those of the original images, a gold standard is available: for the rotation experiments, the references images are simply the original images, and for the translation experiments, the reference images are obtained by translating the original image by four pixels (which requires no interpolation). In order to avoid quantization errors, all computations were carried out with double precision floating-point numbers (12 significant decimals).

\section{$3.2 \quad$ Results}

As can be concluded from the literature, the linear interpolation kernel, $h_{\text {Lin }}$, is by far the most frequently used kernel [7]. Therefore, in this study, our main interest was to investigate the performance of alternative kernels compared to $h_{\text {Lin }}$. To this end, we computed for every test-image and type of experiment, the percentile RMSE of every interpolation kernel compared to $h_{\text {Lin }}$. Since, in all cases, the percentile errors of the five images from a given combination of modality and slice direction were very similar, they were averaged. The results of the translation experiments are presented in Table 3, and those of the rotation experiments in Table 4. For every modality, type of experiment, and kernel extent, only the top-3 best kernels are shown.

\section{Discussion}

The performance of interpolation kernels may be assessed by subjective visual inspection of image quality, after having used the kernels to perform certain resampling operations $[8,5]$. An alternative evaluation approach is to compare the spectral characteristics of the kernels to those of the ideal sinc kernel $[9,10]$, or 


\begin{tabular}{|c|c|c|c|c|c|c|c|c|c|c|c|}
\hline \multirow{3}{*}{$\begin{array}{l}\text { Mod } \\
\text { CT }\end{array}$} & \multirow{3}{*}{$\begin{array}{c}\text { Slc } \\
\operatorname{Tr}\end{array}$} & \multicolumn{10}{|c|}{ Extent } \\
\hline & & \multicolumn{2}{|c|}{$m=1$} & \multicolumn{2}{|c|}{$m=2$} & \multicolumn{2}{|c|}{$m=3$} & \multicolumn{2}{|c|}{$m=4$} & \multicolumn{2}{|c|}{$m=5$} \\
\hline & & $h_{\text {Lin }}$ & 100.0 & $h_{C u b}^{\alpha_{b}}$ & 33.4 & $h_{\text {Kai }}^{\alpha=5}$ & 12.3 & $h_{K a i}^{\alpha=6}$ & 6.3 & $h_{K a i}^{\alpha=7}$ & 4.7 \\
\hline & & $h_{N N}$ & 497.1 & $h_{K a i}^{\alpha=6}$ & 63.6 & $h_{B H 3}$ & 26.7 & $h_{B H 3}$ & 10.4 & $h_{B H 3}$ & 5.6 \\
\hline & & $h_{W e l}$ & 805.4 & $h_{\text {Ham }}$ & 163.0 & $h_{B l a}$ & 28.3 & $h_{B l a}$ & 16.6 & $h_{B H_{4}}$ & 8.6 \\
\hline & $\mathrm{Sa}$ & $h_{\text {Lin }}$ & 100.0 & $h_{C u b}^{\alpha_{b}}$ & 70.1 & $h_{\text {Kai }}^{\alpha=5}$ & 52.0 & $h_{\text {Lan }}$ & 42.7 & $h_{\text {Lan }}$ & 37.5 \\
\hline & & $h_{W e l}$ & 193.9 & $h_{\text {Kai }}^{\alpha=5}$ & 76.2 & $h_{\text {Lan }}$ & 54.0 & $h_{C o s}$ & 42.9 & $h_{C o s}$ & 37.9 \\
\hline & & $h_{N N}$ & 281.1 & $h_{\text {Ham }}$ & 79.2 & $h_{C o s}$ & 54.1 & $h_{K a i}^{\alpha=5}$ & 43.8 & $h_{K a i}^{\alpha=5}$ & 39.0 \\
\hline \multirow[t]{6}{*}{ MR } & $\operatorname{Tr}$ & $h_{\text {Lin }}$ & 100.0 & $h_{C u b}^{\alpha_{b}}$ & 66.9 & $h_{\text {Kai }}^{\alpha=5}$ & 47.1 & $h_{\text {Lan }}$ & 37.8 & $h_{\text {Lan }}$ & 31.5 \\
\hline & & $h_{W e l}$ & 211.2 & $h_{K a i}^{\alpha=5}$ & 74.5 & $h_{\text {Ham }}$ & 49.9 & $h_{K a i}^{\alpha=5}$ & 38.4 & $h_{C o s}$ & 31.9 \\
\hline & & $h_{N N}$ & 310.0 & $h_{\text {Ham }}$ & 78.2 & $h_{\text {Lan }}$ & 50.9 & $h_{C o s}$ & 38.5 & $h_{\text {Kai }}^{\alpha=5}$ & 33.3 \\
\hline & $\mathrm{Sa}$ & $h_{\text {Lin }}$ & 100.0 & $h_{C u b}^{\alpha}$ & 71.7 & $h_{C o s}$ & 55.9 & $h_{\operatorname{Cos}}$ & 46.4 & $h_{\text {Lan }}$ & 41.6 \\
\hline & & $h_{W e l}$ & 162.3 & $h_{\text {Kai }}^{\alpha=5}$ & 79.3 & $h_{\text {Lan }}$ & 56.1 & $h_{\text {Lan }}$ & 46.4 & $h_{C o s}$ & 41.7 \\
\hline & & $h_{\text {Cos }}$ & 230.6 & $h_{W e l}$ & 79.8 & $h_{\text {Kai }}^{\alpha=5}$ & 56.3 & $h_{W e l}$ & 47.8 & $h_{W e l}$ & 42.8 \\
\hline \multirow[t]{6}{*}{ PET } & $\operatorname{Tr}$ & $h_{\text {Lin }}$ & 100.0 & $h_{C u b}^{\alpha_{b}}$ & 36.5 & $h_{\text {Kai }}^{\alpha=5}$ & 21.2 & $h_{K a i}^{\alpha=6}$ & 16.9 & $h_{K a i}^{\alpha=6}$ & 15.3 \\
\hline & & $h_{W e l}$ & 448.6 & $h_{\text {Kai }}^{\alpha=6}$ & 62.5 & $h_{B H 3}$ & 31.4 & $h_{B H 3}$ & 19.8 & $h_{B H 3}$ & 16.4 \\
\hline & & $h_{N N}$ & 542.7 & $h_{\text {Ham }}$ & 90.9 & $h_{B l a}$ & 31.8 & $h_{B l a}$ & 20.9 & $h_{\text {Bla }}$ & 17.0 \\
\hline & $\mathrm{Sa}$ & $h_{\text {Lin }}$ & 100.0 & $h_{C u b}^{\alpha_{b}}$ & 66.5 & $h_{\text {Kai }}^{\alpha=5}$ & 47.9 & $h_{\text {Lan }}$ & 39.2 & $h_{\text {Lan }}$ & 34.6 \\
\hline & & $h_{W e l}$ & 173.3 & $h_{\text {Kai }}^{\alpha=5}$ & 72.0 & $h_{\text {Lan }}$ & 48.7 & $h_{C o s}$ & 39.5 & $h_{C o s}$ & 34.7 \\
\hline & & $h_{C o s}$ & 247.6 & $h_{\text {Ham }}$ & 74.9 & $h_{C o s}$ & 49.0 & $h_{\text {Kai }}^{\alpha=5}$ & 40.4 & $h_{W e l}$ & 35.8 \\
\hline
\end{tabular}

Table 3. The percentile root mean square errors (RMSEs) resulting from the different interpolation kernels in the translation experiments, relative to the RMSEs of the linear interpolation kernel, $h_{\text {Lin }}$. For every extent $(m)$, modality (Mod), and slice direction (Slc), either transversal (Tr) or sagittal (Sa), only the top-3 best kernels are shown.

to compare their abilities to reconstruct certain mathematical test-functions [11]. In the evaluation described in this paper, we have chosen to use a more pragmatic approach, in which the different interpolation kernels are used to apply actual geometrical transformations, which are frequently required in e.g. medical image registration problems. The design of the evaluation was such that true gold standards were available, viz., the original images.

The results of the evaluation allow us to draw some important conclusions. For $m=1$, i.e., a spatial extent of 2 grid points, the best interpolation kernel is the linear interpolation kernel. For $m=2$, i.e., a spatial extent of 4 grid points, the best approach is to use a cubic convolution kernel, although the optimal value for the free parameter, $\alpha$, may differ for different types of images. For the test-images used in this study, cubic convolution resulted in a considerable $(28 \%-75 \%)$ reduction of interpolation errors, as compared to linear interpolation. Even better results ( $44 \%-95 \%$ reduction) were obtained with kernels of larger extent. These latter results showed that, for $m \geqslant 3$, most windowed sinc kernels give better results than piecewise polynomial kernels, although one must 


\begin{tabular}{|c|c|c|c|c|c|c|c|c|c|c|c|}
\hline \multirow{3}{*}{$\begin{array}{l}\text { Mod } \\
\text { CT }\end{array}$} & \multirow{3}{*}{$\begin{array}{l}\text { Slc } \\
\mathrm{Tr}\end{array}$} & \multicolumn{10}{|c|}{ Extent } \\
\hline & & \multicolumn{2}{|c|}{$m=1$} & \multicolumn{2}{|c|}{$m=2$} & \multicolumn{2}{|c|}{$m=3$} & \multicolumn{2}{|c|}{$m=4$} & \multicolumn{2}{|c|}{$m=5$} \\
\hline & & $h_{\text {Lin }}$ & 100.0 & $h_{C u b}^{\alpha_{b}}$ & 24.7 & $h_{\text {Kai }}^{\alpha=5}$ & 10.8 & $h_{K a i}^{\alpha=6}$ & 5.7 & $h_{\text {Kai }}^{\alpha=7}$ & 5.1 \\
\hline & & $h_{N N}$ & 173.6 & $h_{K a i}^{\alpha=6}$ & 56.2 & $h_{B H 3}$ & 21.7 & $h_{B H 3}$ & 9.9 & $h_{B H 3}$ & 5.5 \\
\hline & & $h_{W e l}$ & 997.5 & $h_{\text {Ham }}$ & 247.6 & $h_{Q u i}^{\alpha_{b}}$ & 22.5 & $h_{B H_{4}}$ & 14.0 & $h_{B H 4}$ & 7.0 \\
\hline & $\mathrm{Sa}$ & $h_{\text {Lin }}$ & 100.0 & $h_{C u b}^{\alpha \sim}$ & 58.9 & $h_{\text {Kai }}^{\alpha=5}$ & 45.1 & $\overline{h_{W e l}}$ & 35.4 & $\overline{h_{W e l}}$ & 30.2 \\
\hline & & $h_{N N}$ & 143.1 & $h_{K a i}^{\alpha=6}$ & 76.0 & $h_{W e l}$ & 46.0 & $h_{C o s}$ & 36.5 & $h_{C o s}$ & 30.8 \\
\hline & & $h_{W e l}$ & 240.7 & $h_{\text {Ham }}$ & 83.4 & $h_{C o s}$ & 48.0 & $h_{K a i}^{\alpha=5}$ & 38.6 & $h_{\text {Lan }}$ & 32.1 \\
\hline \multirow[t]{6}{*}{ MR } & $\operatorname{Tr}$ & $h_{\text {Lin }}$ & 100.0 & $h_{C u b}^{\alpha \sim}$ & 59.2 & $h_{\text {Kai }}^{\alpha=5}$ & 43.7 & $h_{W e l}$ & 35.2 & $h_{W e l}$ & 30.3 \\
\hline & & $h_{N N}$ & 153.2 & $h_{K a i}^{\alpha=6}$ & 74.1 & $h_{W e l}$ & 46.1 & $h_{C o s}$ & 36.3 & $h_{C o s}$ & 30.8 \\
\hline & & $h_{W e l}$ & 250.8 & $h_{\text {Ham }}$ & 83.4 & $h_{\operatorname{Cos}}$ & 50.4 & $h_{K a i}^{\alpha=5}$ & 37.8 & $h_{\text {Lan }}$ & 32.0 \\
\hline & $\mathrm{Sa}$ & $h_{\text {Lin }}$ & 100.0 & $h_{C u b}^{\alpha}$ & 62.3 & $h_{W e l}$ & 49.4 & $\overline{h_{W e l}}$ & 40.5 & $h_{W e l}$ & 36.3 \\
\hline & & $h_{N N}$ & 139.3 & $h_{K a i}^{\alpha=5}$ & 76.5 & $h_{K a i}^{\alpha=5}$ & 50.2 & $h_{\text {Cos }}$ & 41.2 & $h_{C o s}$ & 36.7 \\
\hline & & $h_{W e l}$ & 201.0 & $h_{\text {Ham }}$ & 80.8 & $h_{C o s}$ & 51.7 & $h_{\text {Lan }}$ & 42.9 & $h_{\text {Lan }}$ & 37.9 \\
\hline \multirow[t]{6}{*}{ PET } & $\operatorname{Tr}$ & & 100.0 & $h_{C u b}^{\alpha_{b}}$ & 30.1 & $h_{\text {Kai }}^{\alpha=5}$ & 18.3 & $h_{K a i}^{\alpha=6}$ & 15.7 & $h_{\text {Kai }}^{\alpha=7}$ & 14.7 \\
\hline & & $h_{N N}$ & 177.1 & $h_{K a i}^{\alpha=6}$ & 54.2 & $h_{B H 3}$ & 27.6 & $h_{B H 3}$ & 18.2 & $h_{B H 3}$ & 15.5 \\
\hline & & $h_{W e l}$ & 515.7 & $h_{\text {Ham }}$ & 123.3 & $h_{Q u i}^{\alpha_{b}}$ & 28.5 & $h_{B l a}$ & 20.7 & $h_{B l a}$ & 16.8 \\
\hline & $\mathrm{Sa}$ & & 100.0 & $h_{C u b}^{\alpha_{b}}$ & 57.1 & $h_{\text {Kai }}^{\alpha=5}$ & 42.1 & $h_{W e l}$ & 33.5 & $h_{W e l}$ & 29.1 \\
\hline & & $h_{N N}$ & 135.5 & $h_{K a i}^{\alpha=6}$ & 72.6 & $h_{W e l}$ & 44.3 & $h_{C o s}$ & 34.8 & $h_{C o s}$ & 29.7 \\
\hline & & $h_{W e l}$ & 231.5 & $h_{\text {Ham }}$ & & $h_{C o s}$ & 48.1 & $h_{K a i}^{\alpha=5}$ & 36.5 & $h_{\text {Lan }}$ & 30.9 \\
\hline
\end{tabular}

Table 4 . The percentile root mean square errors (RMSEs) resulting from the different interpolation kernels in the rotation experiments, relative to the RMSEs of the linear interpolation kernel, $h_{\text {Lin }}$. For every extent $(m)$, modality (Mod), and slice direction (Slc), either transversal (Tr) or sagittal (Sa), only the top-3 best kernels are shown.

be very careful in choosing a window function. Of the window functions incorporated in this study, the Welch, Cosine, Lanczos, and Kaiser windows appeared to be the best. It was also concluded that a truncated sinc kernel (resulting from applying a rectangular window), was one of the worst performing kernels. Finally, we notice that due to the anisotropic nature of 3-D medical datasets, the through-plane interpolation errors were considerably larger than the in-plane errors. This implies that through-plane interpolation usually requires larger kernels in order for the errors to be comparable to in-plane linear interpolation errors.

\section{Conclusions}

In this paper we have presented the results of a quantitative comparison of sincapproximating kernels for medical image interpolation. The evaluation involved the application of several geometrical transformations (rotations and subpixel translations) to a number of medical images from different modalities (CT, MR, 
and PET), using the different interpolation kernels, and by comparing the resulting grey-value errors to those resulting from linear interpolation. A total of 84 different kernels were evaluated, with spatial extents ranging from 2 to 10 grid points in each dimension.

The results of the evaluation show very clearly that, of the kernels with a spatial extent of 2 grid points, the linear interpolation kernel is the best. Of the kernels with an extent of 4 grid points, the cubic convolution kernel is the best ( $28 \%-75 \%$ reduction of the errors as compared to linear interpolation). Even better results (44\% - 95\% reduction) were obtained with kernels of larger extent, notably the Welch, Cosine, Lanczos, and Kaiser windowed sinc kernels. The truncated sinc kernel was one of the worst performing kernels.

\section{Acknowledgment}

The images used in the experiments described in this paper were obtained from Vanderbilt University, and were originally used in the project "Evaluation of Retrospective Image Registration", National Institutes of Health, Project Number: 1 R01 NS33926-01, Principal Investigator: Prof. Dr. J. Michael Fitzpatrick, Vanderbilt University, Nashville, TN, USA.

\section{References}

1. J. P. W. Pluim, J. B. A. Maintz, \& M. A. Viergever, "Interpolation artefacts in mutual information based image registration", Computer Vision and Image Understanding, 1999. In Press. 210

2. J. L. Ostuni, A. K. S. Santha, V. S. Mattay, D. R. Weinberger, R. L. Levin, \& J. A. Frank, "Analysis of interpolation effects in the reslicing of functional MR images", Journal of Computer Assisted Tomography, vol. 21, no. 5, pp. 803-810, 1997. 210

3. S. Schreiner, C. B. Paschal, \& R. L. Galloway, "Comparison of projection algorithms used for the construction of maximum intensity projection images", Journal of Computer Assisted Tomography, vol. 20, no. 1, pp. 56-67, 1996. 210

4. E. H. W. Meijering, K. J. Zuiderveld, \& M. A. Viergever, "Image reconstruction by convolution with symmetrical piecewise $n$ th-order polynomial kernels", IEEE Transactions on Image Processing, vol. 8, no. 2, pp. 192-201, 1999. 211

5. G. Wolberg, Digital Image Warping, IEEE Computer Society Press, Washington, USA, 1990. 211, 213, 214

6. F. J. Harris, "On the use of windows for harmonic analysis with the discrete Fourier transform", Proceedings of the IEEE, vol. 66, no. 1, pp. 51-83, 1978. 213

7. G. J. Grevera \& J. K. Udupa, "An objective comparison of 3-D image interpolation methods", IEEE Transactions on Medical Imaging, vol. 17, no. 4, pp. 642-652, 1998. 214

8. J. A. Parker, R. V. Kenyon, \& D. E. Troxel, "Comparison of interpolating methods for image resampling", IEEE Transactions on Medical Imaging, vol. 2, no. 1, pp. 31-39, 1983. 214

9. S. K. Park \& R. A. Schowengerdt, "Image reconstruction by parametric cubic convolution", Computer Vision, Graphics and Image Processing, vol. 23, no. 3, pp. 258-272, 1983. 214 
10. E. Maeland, "On the comparison of interpolation methods", IEEE Transactions on Medical Imaging, vol. 7, no. 3, pp. 213-217, 1988. 214

11. R. G. Keys, "Cubic convolution interpolation for digital image processing", IEEE Transactions on Acoustics, Speech, and Signal Processing, vol. 29, no. 6, pp. 11531160, 1981. 215 